\title{
Influence of ethylene oxide gas treatment on the in vitro degradation behavior of dermal sheep collagen
}

\author{
L. H. H. Olde Damink, ${ }^{1}$ P. J. Dijkstra, ${ }^{1}$ M. J. A. Van Luyn, ${ }^{2}$ P. B. Van Wachem, ${ }^{2}$ P. Nieuwenhuis, ${ }^{2}$ and \\ J. Feijen ${ }^{1, *}$ \\ ${ }^{1}$ Department of Chemical Technology, University of Twente, P.O. Box 217, 7500 AE, Enschede; ${ }^{2}$ Department of \\ Histology and Cell Biology, University of Groningen, Oostersingel 69/2, 9713 EZ, Groningen. The Netherlands
}

The influence of ethylene oxide gas treatment on the in vitro degradation behavior of noncrosslinked, glutaraldehyde crosslinked or hexamethylene diisocyanate crosslinked dermal sheep collagen (DSC) using bacterial collagenase is described. The results obtained were compared with the degradation behavior of either nonsterilized or $\gamma$-sterilized DSC. Upon ethylene oxide sterilization, reaction of ethylene oxide with the free amine groups of DSC occurred, which resulted in a decreased helix stability, as indicated by a lowering of the shrinkage temperature of all three types of DSC. Except for the low strain modulus the mechanical properties of the ethylene oxide sterilized materials were not significantly altered. $\gamma$-Sterilization induced chain scission in all three types of DSC, resulting in a decrease of both the tensile strength and the high strain modulus of noncrosslinked and crosslinked DSC. When exposed to a solution of bacterial collagenase, ethylene oxide sterilized materials had a lower rate of degradation compared with nonsterilized DSC. This has been explained by a reduced adsorption of the collagenase onto the collagen matrix as a result of the introduction of pendant $\mathrm{N}$-2-hydroxy ethyl groups. () 1995 John Wiley \& Sons, Inc.

\section{INTRODUCTION}

$\gamma$-Irradiation is frequently used to sterilize collagenbased biomaterials, ${ }^{1}$ mainly for its high efficiency and the possibility of packaging and sealing before sterilization. A dose of $25 \mathrm{kGy}$ ( $2.5 \mathrm{Mrad}$ ) is regarded to achieve complete sterilization. ${ }^{2}$ Although it was suggested that the properties of collagen-based biomaterials are not altered using this dose, ${ }^{3}$ several studies have reported that high-energy irradiation can interact with collagen-based materials even at doses as low as $5 \mathrm{kGy}^{4,5}$ It was shown that $\gamma$-irradiation induced chain cleavage in soluble tropocollagen, ${ }^{6}$ reconstituted fibrils, ${ }^{6}$ or the fibrous collagen network obtained from purified dermis., ${ }^{4,5}$ Because of the chain cleavages, the triple-helix stability and the tensile strength of the materials decreased. Furthermore, $\gamma$-sterilization increased the rate of degradation, as determined both in vitro ${ }^{6}$ and in vivo. ${ }^{8}$ The decrease in tensile strength and the increase in bioresorption induced by $\gamma$-sterilization are disadvantageous when

*To whom correspondence should be addressed. collagen-based materials are used in load-bearing applications.

Ethylene oxide gas treatment has been used as an alternative method to sterilize collagen-based biomaterials. ${ }^{1}$ It is reported that ethylene oxide sterilization does not affect the tensile strength of collagen-based biomaterials. ${ }^{9}$ However, it is known that during sterilization of collagen, ethylene oxide molecules can react with the amine groups of lysine or hydroxylysine ${ }^{3}$ and will decrease the triple-helix stability. To our knowledge, no reports are available on the influence of ethylene oxide sterilization on the in vitro or in vivo degradation of collagen-based materials.

Here, we report on the influence of ethylene oxide sterilization on the in vitro degradation of dermal sheep collagen (DSC). DSC was crosslinked either with glutaraldehyde or with hexamethylene diisocyanate, and the resulting materials were exposed to an aqueous bacterial collagenase solution. ${ }^{10}$ The degradation behavior of ethylene oxide-sterilized materials was compared with the degradation behavior of either nonsterilized or $\gamma$-sterilized noncrosslinked DSC (N-DSC), glutaraldehyde-crosslinked DSC (G-DSC), and hexamethylene diisocyanate crosslinked DSC $(\mathrm{H}-$ DSC). 


\section{MATERIALS AND METHODS}

Dermal sheep collagen was crosslinked with glutaraldehyde (GA) or hexamethylene diisocyanate (HMDIC) to give G-DSC or H-DSC, respectively. ${ }^{10}$

The shrinkage temperature (Ts) and free amine group content of sterilized or nonsterilized N-DSC, G-DSC, or H-DSC samples were determined as described previously. ${ }^{10}$ Stress-strain curves were determined by uniaxial measurements using an Instron mechanical tester. Samples used to study the influence of sterilization on the mechanical properties were always taken from the IUP/2 sampling area parallel to the backbone and were either sterilized with $\gamma$-irradiation or ethylene oxide or kept as control.

\section{Sterilization procedures}

Noncrosslinked or crosslinked DSC samples were air-dried for at least 1 day before either $\gamma$ - or ethylene oxide sterilization procedures were conducted. DSC samples were sealed in plastic bags before $\gamma$-sterilization and were exposed to $25 \mathrm{kGy}(2.5 \mathrm{Mrad})$ gamma irradiation from a ${ }^{60} \mathrm{Co}$ source (Gammaster, Ede, The Netherlands). Ethylene oxide gas treatment was performed at the University Hospital of the University of Groningen (Groningen, The Netherlands) using a standard ethylene oxide sterilization apparatus ( $\mathrm{Fa}$ Hubert, Sneek, The Netherlands). The samples were exposed to a $100 \%$ ethylene oxide atmosphere at a relative humidity of $70 \%$ for $5 \mathrm{~h}$ at $55^{\circ} \mathrm{C}$. The samples were aerated with warm air flow at atmospheric pressures for at least $48 \mathrm{~h}$ to remove residual ethylene oxide from the DSC matrix, and stored in laminated bags until use.

\section{In vitro degradation}

The weight loss and tensile strength upon degradation of nonsterilized and sterilized N-DSC, G-DSC, and $\mathrm{H}$-DSC were determined using the general procedure as described previously. ${ }^{10}$ Bacterial collagenase from Clostridium histolyticum (EC 3.4.24.3, Sigma Chemical Company, St Louis, MO) with a collagenase activity of $255 \mathrm{U} / \mathrm{mg}$ was used.

\section{RESULTS}

\section{Sterilization}

Ts and free amine group content

The results obtained from the Ts measurements and the free amine group content determinations of sterilized and nonsterilized N-DSC, G-DSC, and $\mathrm{H}$-DSC are presented in Table I. Crosslinking of $\mathrm{N}$-DSC with HMDIC increased the Ts from $49^{\circ} \mathrm{C}$ for $\mathrm{N}$-DSC to $67^{\circ} \mathrm{C}$, whereas crosslinking with GA increased the Ts to $72^{\circ} \mathrm{C}$. The free amine group content expressed as number of free amine groups present per 1000 amino acid residues $(n / 1000)$ of N-DSC was $30.5 \pm 1.1$ per 1000 amino acid residues. Crosslinking of N-DSC with HMDIC or GA through the pendant amino groups of amino acid residues such as lysine and hydroxylysine resulted in a free amine group content of $19.1 \pm 0.2 / 1000$ for H-DSC and 10.6 $\pm 0.1 /$ 1000 for G-DSC.

Both $\gamma$ - and ethylene oxide sterilization resulted in lower values of Ts. After $\gamma$-sterilization the Ts of $\mathrm{N}$-DSC was $41^{\circ} \mathrm{C}$, whereas for H-DSC and G-DSC, Ts values of 49 and $55^{\circ} \mathrm{C}$, respectively, were found. Contrary to $\gamma$-sterilization, ethylene oxide sterilization resulted only in slightly lower values of Ts. Upon ethylene oxide sterilization of N-DSC a Ts of $46^{\circ} \mathrm{C}$ was found, whereas for H-DSC and G-DSC these values were 62 and $65^{\circ} \mathrm{C}$, respectively.

$\gamma$-Sterilization of N-DSC, G-DSC, and H-DSC samples resulted in slightly higher free amine group contents compared with the nonsterilized samples. The initial values determined for N-DSC (31/1000), H-DSC $(19 / 1000)$, and G-DSC $(10.6 / 1000)$ were increased by $\gamma$-sterilization to $36 / 1000,22 / 1000$, and $11.0 / 1000$, respectively. Contrary to $\gamma$-irradiation, ethylene oxide sterilization of N-DSC, H-DSC, and G-DSC resulted in very low values for the free amine group content. After ethylene oxide sterilization, a free amine group content of five to eight amine groups per 1000 amino acids was observed for the DSC samples.

TABLE I

Shrinkage Temperature and Free Amine Group Content of Nonsterilized and Sterlized N-DSC, H-DSC, and G-DSC

\begin{tabular}{llcr} 
Sample & $\begin{array}{c}\text { Sterilization } \\
\text { Procedure } \\
\text { Used }\end{array}$ & $\begin{array}{c}\text { Shrinkage } \\
\text { Temperature } \\
\left({ }^{\circ} \mathrm{C}\right)\end{array}$ & $\begin{array}{c}\text { Amine Group } \\
\text { Content } \\
(n / 1000)\end{array}$ \\
\hline N-DSC $^{*}$ & & $49.2 \pm 0.3$ & $30.5 \pm 1.1$ \\
& Gamma & $41.2 \pm 1.0$ & $35.8 \pm 0.1$ \\
H-DSC & Ethylene oxide & $46.1 \pm 0.3$ & $8.4 \pm 0.1$ \\
& Gamma & $67.0 \pm 0.4$ & $19.1 \pm 0.2$ \\
& Ethylene oxide & $61.9 \pm 0.4$ & $6.0 \pm 0.7$ \\
G-DSC & & $71.6 \pm 0.5$ & $10.6 \pm 0.1$ \\
& Gamma & $55.4 \pm 0.7$ & $11.0 \pm 0.1$ \\
& Ethylene oxide & $65.0 \pm 0.4$ & $5.4 \pm 0.1$ \\
\hline
\end{tabular}

*Noncrosslinked DSC.

${ }^{\dagger}$ Hexamethylene diisocyanate crosslinked DSC.

‡Glutaraldehyde-crosslinked DSC.

The amine group content of DSC is expressed as the number of amine groups present per 1000 amino acids $(n=3)$. 


\section{Mechanical properties}

Table II presents the results from mechanical measurements of both $\gamma$ - and ethylene oxide-sterilized N-DSC, G-DSC, and H-DSC, respectively. $\gamma$-Sterilization caused a large decrease in the mechanical properties of the N-DSC samples. The tensile strength decreased from 12.3 to $0.6 \mathrm{MPa}$ and the elongation at break decreased from 191 to $72 \%$. The stress-strain curve of $\gamma$-sterilized N-DSC had only one modulus, which was equal to $1.3 \mathrm{MPa}$. For G-DSC and H-DSC the effect of $\gamma$-irradiation on the mechanical properties was less pronounced compared with N-DSC. The tensile strength of H-DSC samples decreased from 16.3 to $7.4 \mathrm{MPa}$, whereas the high strain modulus decreased from 12.3 to $6.0 \mathrm{MPa}$. For G-DSC a decrease in tensile strength from 14.0 to $10.2 \mathrm{MPa}$ and a decrease in high strain modulus from 9.6 to $7.0 \mathrm{MPa}$ were observed. No significant changes in elongation at break, low strain modulus, and elongation at alignment were detected after $\gamma$-sterilization of G-DSC and H-DSC.

In contrast to $\gamma$-sterilization, ethylene oxide sterilization caused an increase in low strain modulus for all materials. The low strain modulus of N-DSC increased from 1.3 to $2.9 \mathrm{MPa}$, whereas for $\mathrm{H}$-DSC an increase from 3.6 to $4.7 \mathrm{MPa}$ was found. The low strain modulus of G-DSC increased from 2.9 to 3.7 $\mathrm{MPa}$. No significant differences were found for the tensile strength, elongation at alignment, high strain modulus, and elongation at break after ethylene oxide sterilization of N-DSC, G-DSC, and H-DSC, respectively.

\section{In vitro degradation}

To study the influence of either $\gamma$ - or ethylene oxide sterilization on in vitro degradation, sterilized and nonsterilized N-DSC, G-DSC, and H-DSC samples were exposed to bacterial collagenase, and the change in weight and tensile strength of the samples was monitored as a function of degradation time. In a previous study ${ }^{10}$ it was shown that the tensile strength of noncrosslinked and GA- or HMDIC-crosslinked DSC samples is more sensitive to degradation than the high strain modulus or the elongation at break. Therefore, only the decrease in tensile strength during degradation was determined. The change in weight and tensile strength of the samples is expressed as the percentage of the original value, respectively, of weight and tensile strength left.

Figure 1 presents the percentage of weight-loss of $\mathrm{N}$-DSC samples as a function of degradation time. The rate of weight loss as determined for nonsterilized N-DSC was $50 \% / h$. Compared with nonsterilized N-DSC, this rate was increased to $94 \% / \mathrm{h}$ for $\gamma$-sterilized N-DSC, but the rate of weight loss of ethylene oxide sterilized N-DSC was decreased to $32 \%$ / $h$. Because all partially degraded N-DSC samples behaved like denatured collagen, no cross-sectional area of the test specimens could be measured, and consequently, the tensile strength could not be determined. Therefore, only the time at which the DSC samples completely disintegrated when the degradation tube was vigorously shaken was determined. Complete disintegration of either nonsterilized, $\gamma$-irradiated N-DSC, or ethylene oxide-sterilized N-DSC was observed after a 45-min, 35- $\mathrm{min}$, and 65-min degradation period, respectively.

The changes in weight and tensile strength upon degradation of G-DSC are presented in Figure 2a and b. The rate of weight loss of G-DSC samples was much lower compared with the corresponding $\mathrm{N}$-DSC samples. $\gamma$-Irradiation increased the rate of weight loss from $0.3 \% / \mathrm{h}$ for nonsterilized G-DSC to $1.6 \% / \mathrm{h}$ for $\gamma$-sterilized G-DSC (Fig. 2a), whereas for ethylene oxide-sterilized G-DSC this rate was reduced to $0.1 \% / \mathrm{h}$. The changes in tensile strength of

TABLE II

Mechanical Properties of Nonsterilized and Sterilized N-DSC, H-DSC, and G-DSC Samples

\begin{tabular}{|c|c|c|c|c|c|c|}
\hline Sample & $\begin{array}{l}\text { Sterilization } \\
\text { Procedure }\end{array}$ & $\begin{array}{c}\text { Tensile Strength } \\
(\mathrm{MPa})\end{array}$ & $\begin{array}{l}\text { Elongation at } \\
\text { Alignment (\%) }\end{array}$ & $\begin{array}{c}\text { Elongation at } \\
\text { Break }(\%)\end{array}$ & $\begin{array}{c}\text { Low Strain } \\
\text { Modulus (MPa) }\end{array}$ & $\begin{array}{c}\text { High Strain } \\
\text { Modulus (MPa) }\end{array}$ \\
\hline \multirow[t]{3}{*}{ N-DSC ${ }^{*}$} & & $12.3 \pm 0.5$ & $65 \pm 3$ & $191 \pm 12$ & $1.3 \pm 0.2$ & $9.8 \pm 1.7$ \\
\hline & Gamma & $0.6 \pm 0.1$ & & $72 \pm 5$ & & $1.3 \pm 0.1$ \\
\hline & Ethylene oxide & $14.4 \pm 0.4$ & $73 \pm 9$ & $211 \pm 17$ & $2.9 \pm 0.3$ & $9.6 \pm 1.1$ \\
\hline \multirow[t]{3}{*}{ H-DSC ${ }^{\dagger}$} & & $16.3 \pm 0.7$ & $78 \pm 3$ & $198 \pm 6$ & $3.6 \pm 0.1$ & $12.3 \pm 1.3$ \\
\hline & Gamma & $7.4 \pm 0.3$ & $71 \pm 6$ & $172 \pm 10$ & $3.0 \pm 0.3$ & $6.0 \pm 0.3$ \\
\hline & Ethylene oxide & $16.4 \pm 0.8$ & $75 \pm 2$ & $185 \pm 6$ & $4.7 \pm 0.5$ & $12.8 \pm 0.8$ \\
\hline \multirow[t]{3}{*}{ G-DSC ${ }^{\ddagger}$} & & $14.0 \pm 0.7$ & $76 \pm 4$ & $204 \pm 8$ & $2.9 \pm 0.2$ & $9.6 \pm 0.5$ \\
\hline & Gamma & $10.2 \pm 0.3$ & $70 \pm 7$ & $196 \pm 12$ & $2.9 \pm 0.1$ & $7.0 \pm 0.4$ \\
\hline & Ethylene oxide & $14.7 \pm 0.2$ & $73 \pm 8$ & $213 \pm 6$ & $3.7 \pm 0.3$ & $9.0 \pm 0.5$ \\
\hline
\end{tabular}

*Noncrosslinked DSC.

${ }^{+}$Hexamethylene diisocyanate crosslinked DSC.

‡Glutaraldehyde-crosslinked DSC.

All mechanical properties are measured in fivefold and are given as mean \pm standard deviation. 


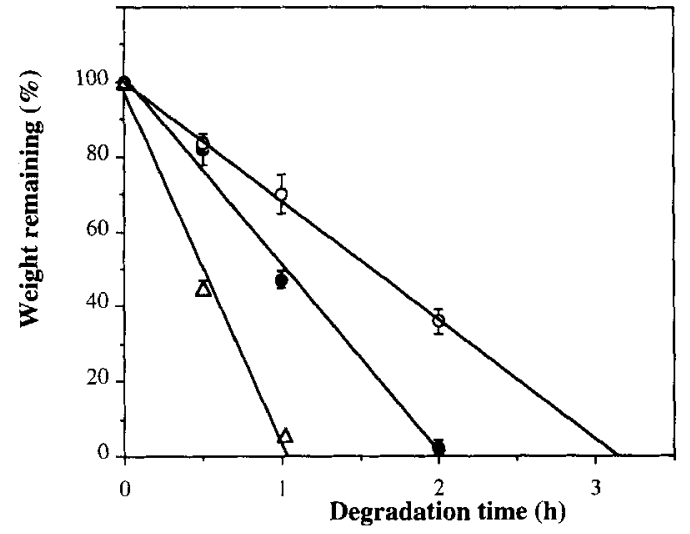

Figure 1. Change in weight as a function of degradation time during exposure of nonsterilized (closed circles), $\gamma$-irradiated (open triangles), and ethylene oxide gas-treated (open circles) N-DSC to bacterial collagenase ( $n=5, \pm$ SD).
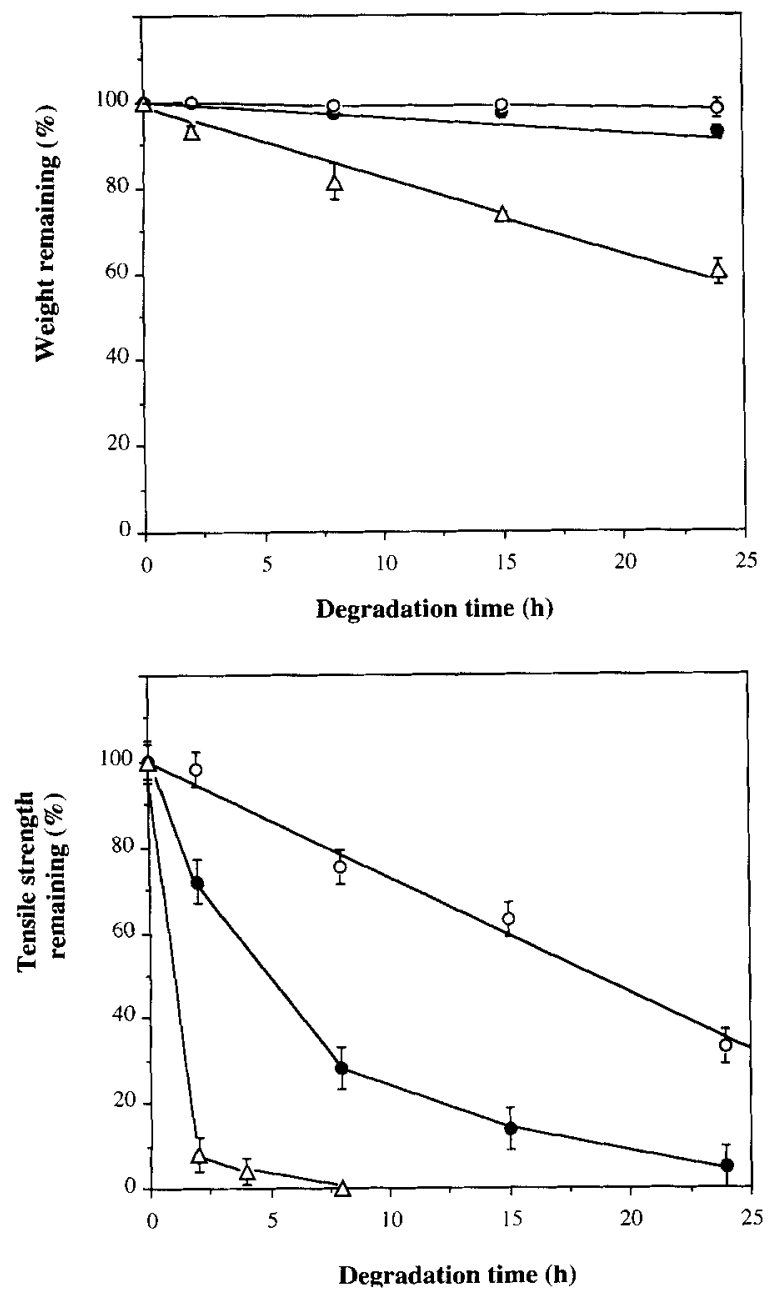

Figure 2. Change in weight (a) and change in tensile strength (b) as a function of degradation time during exposure of nonsterilized (closed circles), $\gamma$-irradiated (open triangles), and ethylene oxide gas-treated (open circles) G-DSC to bacterial collagenase $(n=5, \pm S D)$. nonsterilized and sterilized G-DSC during degradation are presented in Figure $2 b$. Although for nonsterilized G-DSC only $5 \%$ of the initial tensile strength was left after a $24-h$ degradation period, ethylene oxide-sterilized G-DSC retained $33 \%$ of the initial tensile strength after the same time period. No tensile strength could be measured after an 8-h degradation period for $\gamma$-sterilized G-DSC.

The sterilization methods applied to H-DSC samples resulted in similar trends in the rate of weight loss and remaining tensile strength found for G-DSC samples. Although for nonsterilized H-DSC an initial rate of weight loss of $4.8 \%$ /h was observed (Fig. 3a), $\gamma$-irradiation increased this rate to $24 \% / \mathrm{h}$ and ethylene oxide sterilization decreased this rate to $2.2 \% / \mathrm{h}$. The influence of sterilization on the change in tensile strength during degradation is presented in Figure $3 \mathrm{~b}$. The fastest decrease in tensile strength was ob-
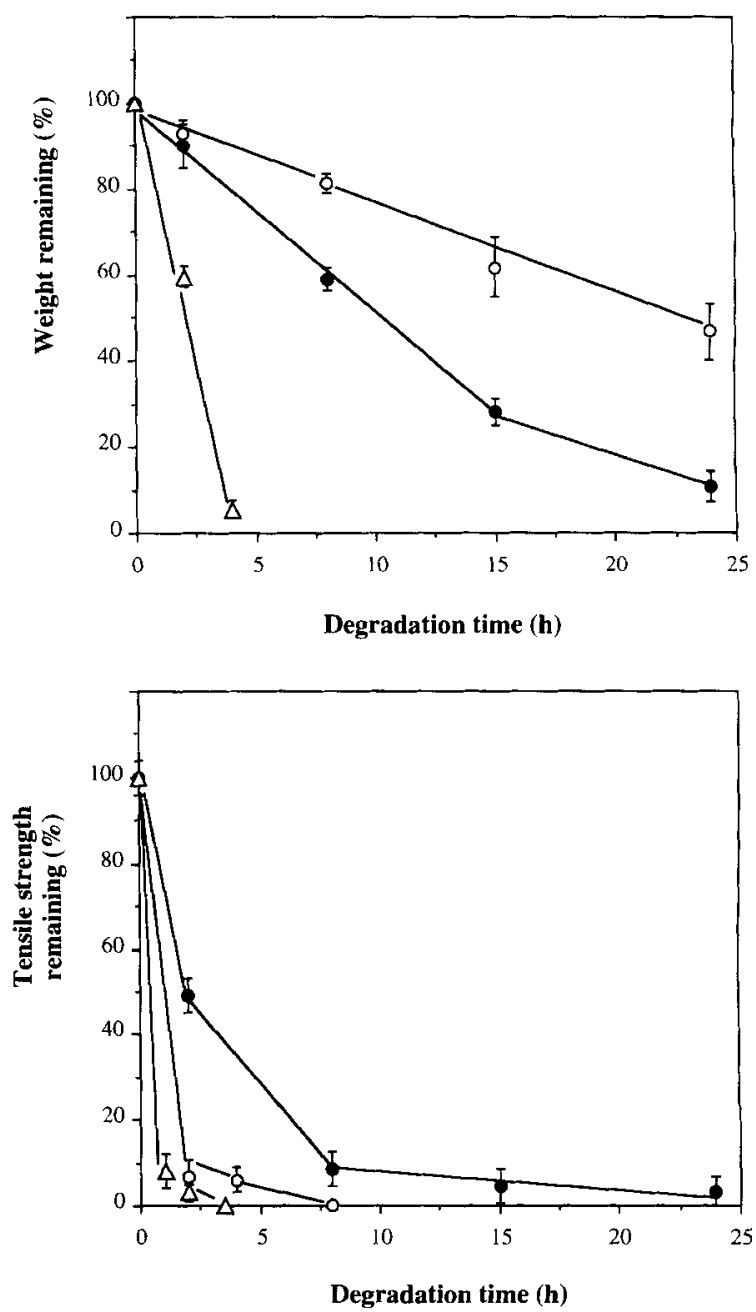

Figure 3. Change in weight (a) and change in tensile strength (b) as a function of degradation time during exposure of nonsterilized (closed circles), $\gamma$-irradiated (open triangles), and ethylene oxide gas-treated (open circles) $\mathrm{H}$-DSC to bacterial collagenase $(n=5, \pm \mathrm{SD})$. 
served for $\boldsymbol{\gamma}$-sterilized H-DSC. No tensile strength was determined for this material after a 3.5-h degradation period. For nonsterilized H-DSC no tensile strength was determined after an 8 -h degradation period. Ethylene oxide-sterilized H-DSC had only $3 \%$ of the initial tensile strength left after a 24-h degradation period.

\section{DISCUSSION}

An ideal sterilization procedure will free collagenbased biomaterials of all viable microorganisms without inducing major changes in the mechanical properties and the degradation behavior of the material. Although $\gamma$-irradiation is frequently used for this purpose, ${ }^{1}$ previous studies showed that $\gamma$-sterilization decreases the tensile strength ${ }^{4,5,7}$ and increases the rate of bioresorption ${ }^{6,8}$ of collagen-based biomaterials.

In contrast, ethylene oxide gas treatment as an alternative sterilization method does not induce changes in the tensile strength of the materials. ${ }^{9}$ Therefore, ethylene oxide sterilization appears to be advantageous when collagen-based biomaterials are used in a load-bearing application such as abdominal wall repair. Especially in such an application, it is important to maintain the mechanical properties of the materials during the desired implantation period. However, when ethylene oxide gas treatment is used as a sterilization method, the presence of residual ethylene oxide may result in a cytotoxic action toward cells or tissue after implantation. ${ }^{11-13}$ The materials used in these studies did not induce any cytotoxic reaction toward cells or tissues that could be associated with the possible presence and release of residual ethylene oxide.

Previously, we described the crosslinking of DSC with either $\mathrm{GA}^{14}$ or $\mathrm{HMDIC},{ }^{15}$ and the in vitro degradation behavior of the resulting materials. ${ }^{10}$ The results obtained revealed that G-DSC had a higher Ts compared with H-DSC. The Ts values presented in Table I reflect the results reported previously. Although this suggests that G-DSC samples have a higher degree of crosslinking than H-DSC, care must be taken directly to relate the degree of crosslinking of collagen samples to their Ts values when these samples are crosslinked with different reagents. The Ts may not only be influenced by the degree of crosslinking of the samples, but also by the type of crosslink introduced.

When exposed to bacterial collagenase, both nonsterilized G-DSC samples and H-DSC samples were more resistant toward degradation than N-DSC, as indicated by a much lower rate of weight loss. ${ }^{10}$ Among the mechanical properties of the crosslinked materials, such as high strain modulus and elongation at break, the tensile strength was most sensitive toward degradation. A slower decrease in tensile strength, and therefore a higher resistance against bacterial collagenase, was observed for the G-DSC samples, which suggested that these materials had a higher degree of crosslinking compared with H-DSC samples.

Treatment of the N-DSC, G-DSC, or H-DSC samples with ethylene oxide gas decreased the free amine group content of the samples (Table I). This decrease results from the reaction of ethylene oxide with amine groups from lysine or hydroxylysine residues. ${ }^{1,3}$ The decrease in Ts of the materials (Table I) as observed by the introduction of $\mathrm{N}$-2-hydroxy ethyl groups possibly destabilizes the tertiary and quarternary structure. The reaction between the epoxide groups and the amine groups has been used for the crosslinking of collagen with polyepoxy fixatives. ${ }^{16}$

Chain scission is the most important phenomenon that occurs when $\gamma$-irradiation is used for the sterilization of collagen-based biomaterials. ${ }^{4,6,7}$ When either N-DSC, G-DSC, or H-DSC samples were subjected to $\gamma$-irradiation using a dose of $25 \mathrm{kGy}$ (Table I), lower values for the Ts of the materials compared with the nonsterilized materials were found. This indicates that the stability of the tertiary and quaternary structure of the dermal sheep collagen decreased by chain scission. Moreover, the free amine group content of all DSC samples increased, which is a result of the generation of amine groups due to scission of amide bonds of the polypeptide chains. ${ }^{6}$

The chain scission induced during $\gamma$-irradiation changed the mechanical properties of N-DSC, G-DSC, and H-DSC samples to a large extent (Table II). Especially for N-DSC, a large decrease in tensile strength and high strain modulus was observed. The influence of $\gamma$-irradiation on the tensile strength and the high strain modulus of G-DSC and H-DSC samples was less pronounced as observed for N-DSC samples. ${ }^{7}$ Contrary to the effect of chain scissions as induced by $\gamma$-irradiation, the reaction of amine groups present in N-DSC, G-DSC, and H-DSC samples with ethylene oxide obviously does not influence the mechanical properties of the materials. No major differences in the mechanical properties of nonsterilized and ethylene oxide-sterilized N-DSC, G-DSC, and H-DSC samples were found (Table II). Only a slight increase in the low strain modulus was found for all three materials.

Both the rate of weight loss and the change in tensile strength of ethylene oxide sterilized N-DSC (Fig. 1), G-DSC (Fig. 2a and b), and H-DSC (Fig. 3a and b) during exposure to bacterial collagenase were decreased compared with the nonsterilized materials. The decrease in rate of degradation observed for ethylene oxide sterilized DSC may be a result of a reduc- 
tion of recognizable adsorption or cleavage sites for the bacterial collagenase resulting from the introduction of $\mathrm{N}$-2-hydroxy ethyl groups. Bacterial collagenase cleaves the polypeptide chain between $X$ and Gly in the peptide sequence Pro-X-Gly-Pro- $Y^{17}$ where $X$ and $Y$ are predominantly apolar amino acid residues. ${ }^{18}$ Therefore, it becomes likely that reaction of lysine residues with ethylene oxide changes the adsorption sites for the collagenase. This eventually results in a smaller number of enzymatic cleavages in time, thus decreasing the rate of degradation. Similar results have been observed after acetylation or succinilation of lysine and hydroxylysine amine groups of collagen in the degradation using enzymes such as trypsine. ${ }^{19}$

On the other hand, side-chain modification also reduces the triple-helix stability, which would make the DSC matrix more susceptible to enzymatic breakdown. The increased resistance against degradation of ethylene oxide-sterilized N-DSC, G-DSC, and $\mathrm{H}$-DSC indicates that changes in the adsorption site of the enzyme are more prominent than the ethylene oxide-induced destabilization of the DSC matrix.

Both the rate of weight loss and the decrease in tensile strength during degradation were fastest for $\gamma$-sterilized DSC samples (Fig. 1, 2, and 3). The increased susceptibility toward enzymatic degradation of the materials after $\gamma$-sterilization is caused by the introduction of chain scissions in the DSC matrix. ${ }^{8}$ This makes the DSC matrix more accessible for collagenase, and will increase the degradation rate. Furthermore, because of chain scissions, the number of enzymatic scissions necessary to induce weight loss becomes lower. This also accounts for the faster change in tensile strength. ${ }^{10}$

Although the degradation of collagen-based materials in vitro may not be extrapolated directly to the in vivo situation, ${ }^{20}$ the action of enzymes such as mammal collagenases or cathepsins may also be effected by the amino acid side-chain modification during ethylene oxide sterilization. This may result in materials having a persistent tensile strength during prolonged implantation periods, which is advantageous when collagen-based biomaterials are applied in load-bearing applications.

\section{CONCLUSIONS}

Ethylene oxide sterilization oxide sterilization of N-DSC, G-DSC, and H-DSC improved the resistance against degradation by bacterial collagenase of the materials compared with nonsterilized DSC. During ethylene oxide sterilization reaction of ethylene oxide molecules with the amine groups of all types of DSC occurred. The introduction of N-2-hydroxy ethyl groups caused no major changes in the mechanical properties of the samples and probably interfered with the adsorption of collagenase. $\gamma$-Sterilized DSC was degraded faster compared with nonsterilized DSC. $\gamma$-Sterilization induced chain scission in the polypeptide chains of all DSC samples. This resulted in a decreased tensile strength and high strain modulus of the samples, and made the samples more susceptible toward degradation.

\section{References}

1. S. D. Bruck and E. P. Mueller, "Radiation sterilization of polymeric implant materials," J. Biomed. Mater. Res., 22, 133-144 (1988).

2. "Radiation sterilization dose-the position of the U.K. panel on gamma and electron irradiation," Radiat. Phys. Chem., 29, 87-88 (1987).

3. M. Chvapil, R. L. Kronenthal, and W. van Winkle Jr., "Medical and surgical applications of collagen," in International Review of Connective Tissue Research, Vol. 6, D. A. Hall and D. S. Jackson, eds., Academic Press, New York, 1973.

4. R. K. Raina, B. K. Wali, and A. M. Wani, "Effect of ${ }^{60} \mathrm{Co}$-gamma radiation on the properties of furs," Radiat. Phys. Chem., 36, 313-315 (1990).

5. C. Kaldirinci, "Effect of gamma radiation on chrometanned sheepskin," Radiochem. Radioanal. Lett., 53, 6168 (1982).

6. D. T. Cheung, N. Perelman, D. Tong, and M. E. Nimni, "The effect of $\gamma$-irradiation on collagen molecules, isolated $\alpha$-chains, and crosslinked native fibers," J. Biomed. Mater. Res., 24, 581-589 (1990).

7. T. M. van Gulik and P. J. Klopper, "Studies on the shrinkage temperature and mechanical properties of gamma-irradiated sheepskin splits," J. Soc. Leather Technol. Chem., 71, 75-79 (1986).

8. M. Chvapil, J. A. Owen, and D. S. Clark, "Effect of collagen crosslinking on the rate of resorption of implanted collagen tubing in rabbits," J. Biomed. Mater. Res., 11, 297-314 (1977).

9. K. Heicken and H. Bellinger, "Die Sterilisation von Catgut mit gasförmigem Athylenoxyd," Zentralblatt für Bakteriologie Abt. I., Org., 173, 113-140 (1960).

10. L. H. H. Olde Damink, H. ten Hoopen, P. J. Dijkstra, M. I. A. van Luyn, P. B. van Wachem, P. Nieuwenhuis, and J. Feijen, "Changes in mechanical properties of dermal sheep collagen during in vitro degradation," J. Biomed. Mater. Res., 29, 139-147 (1995).

11. P. Vink and K. Pleijsier, "Aeration of ethylene oxidesterilized polymers," Biomaterials, 7, 225-230 (1986).

12. E. Saalman, "Infrared spectroscopic study of polymers exposed to ethylene oxide," Biomaterials 6, 225230 (1985).

13. I. P. Matthews, C. Gibson, and A. H. Samuel, "Enhancement of the kinetics of the aeration of ethylene oxide sterilized polymers using microwave radiation," J. Biomed. Mater. Res., 23, 143-156 (1989).

14. L. H. H. Olde Damink, P. J. Dijkstra, M. J. A. van Luyn, P. B. van Wachem, P. Nieuwenhuis, and J. Feijen, "Glutaraldehyde as a crosslinking agent for dermal sheep collagen," Mat. Med., submitted.

15. L. H. H. Olde Damink, P. J. Dijkstra, M. J. A. van Luyn, P. B. van Wachem, P. Nieuwenhuis, and J. Fe- 
ijen, "Crosslinking of dermal sheep collagen using hexamethylene diisocyanate," Mat. Med., submitted.

16. R. Tu, C. L. Lu, K. Thyagarajan, E. Wang, H. Nguyen, S. Shen, C. Hata, and R. C. Quijano, "Kinetic study of collagen fixation with polyepoxide fixatives," J. Biomed. Mater. Res., 27, 3-9 (1993).

17. E. Harper, A. Berger, and E. Katchalski, Biopolymers, 11, 1607-1612 (1972).

18. K. H. Stenzel, T. Miyata, and A. L. Rubin, "Collagen as a biomaterial," Ann. Rev. Biophys. Bioeng., 3, 231253 (1974).

19. A. M. Diamond, S. D. Gorham, D. J. Etherington,
J. G. Robertson, and N. D. Light, "The effect of modification on the susceptibility of collagen to proteolysis: I. Chemical modification of amino acid side chains," Matrix, 11, 321-329 (1991).

20. I. V. Yannas, J. F. Burke, C. Huang, and P. L. Gordon "Correlation of in vivo collagen degradation rate with in vitro measurements," J. Biomed. Mater. Res., 9, 623628 (1975).

Received December 28, 1993

Accepted July 11, 1994 